que nos encontramos enfrentados actualmente es la siguiente: bien optar por una filosofia critica que aparecera como una filosofía analitica de la verdad en general, bien optar por un pensamiento crítico que adoptará la forma de una ontologla de nosotros mismos, una ontología de la actualidad; esa forma de filosofia que, desde Hegel a la Escuela de Frankfurt pasando por Nietzsche y Max Weber, ha fundado una forma de reflexión en la que intento trabajar", Saber y verdad, Julia Varela y F. Alvarez Uria (cds.), Madrid, La Piqueta, 1991, p. 207.

48. M. Weber, Prólogo, en La etica protestante y el espiritu del capitalismo, Barcelona, Pentnsula, 1972 .

49. M. Foucault, "Qu'est-ce que les lumières?*, Magrazine Litteraire (abril, 1993), Gallimard, pp. $63-73$.

50. Ibidem, p. 70 .

51. Ibiden.

52. Aunque no es lo que interesa destacar aquí, habra que recordar que el programa global de Foucault de ecrítica genealogica en su diseño y arqueologica en su métodos también puede ser fructifero para el feminismo. Véase para esta cuestion Isaac D. Balbus, *Michel Foucault y cl poder del discurso feministan, en Seyla Benhabib y Drucilla Comella (eds.), Teoria feminista y teorfa critica, op. cit, pp. 169-191.

53. A. Wellmer, wa dialectica de Modernidad y Postmodemidad, Debats, n." 14 (1985), pp, 6787.

54. M. Foucault, op. cit, p. 72 ,

55. I. Kant, Crítica de la razón pum, op. cit., p. 312 .

56. M. Foucault, op. cit., p. 73.

57. Ibidem.

58. H. Brunkhorst, «Adorno: Dialéctica de la Modemidad, Seminaria sobre Adomo, Departamento de Filosofia, Universidad de Valencia (febrero 1992) (trad. cast. de M. Jimenez Redondo).

59. C. Pateman, The Sexual Cottract, California, Stanford University Press, 1988.

60. H. Brunkhorst, "Adorno: Dialéctica de la modemidadm, seminario citado.

61. Ibident.

62. A. Wellmer, sLa Dialéctica de Modemidad y Postmodemidads, art. cit, p. 86.

\title{
Elogio de la Cordura. (Una crítica al criterio de responsabilidad de Susan Wolf)
}

\author{
MIOUEL BELTRAN \\ Università degli Studi di Venezia
}

I

Sabido es que, desde los siglos xvII y XVIII, y en el debate inglés sobre la cuestión del libre albedrío, se avivó una célebre disputa en torno al asi llamado 'principio de las posibilidades alternativas' - según el cual un agente sólo puede ser considerado responsable de sus acciones si, en un sentido absoluto (esto es, simultáneamente), hubiera podido hacer otra cosa que la que efectivamente hace-. Se vieron cnvucltos en dicha disputa, de un modo primordial, los compatibilistas clásicos, desde Hobbes hasta Moore, y aún ahora se libra esa batalla (transferida a Norteamérica) entre quienes abogan de manera extremada por dicho principio -así la recurrida defensa de Chisholm (1967), o la más reciente de Van Inwagen (1983) y quienes lo denuncian como insignificante para la consideración moral - desde el artículo ya clásico de Frankfurt (1969), hasta las incisivas críticas de Dennett (1984a y $b$ ).

Los dos últimos forman parte de esa tendencia racionalista del compatibilismo actual que incluye en su nomina, 
también, a filósofos como Benson (1987), Watson (1975, 1987), Charles Taylor (1976), Hofstadter $(1979,1982)$, y Susan Wolf $(1987,1990)$. Todos ellos arguyen, con mayor o menor fortuna, que lo que de verdad nos interesa cuando nos preocupamos de si un acto es libre y responsable no es precisamente si podríamos haber actuado de otro modo, conque más allá de realizar ataques contra la desenfrenada libertad de indiferencia que pretenden los libertaristas para el agente moral, el compatibilismo actual se ocupa de redefinir las condiciones de la auto-determinación, que pueden ser tachadas como de irremediablemente deficientes en las argumentaciones compatibilistas anteriores a la canónica formulación hecha por Kant del auto-control.

A este respecto, Schoeman (1987) afirma que «las reflexiones sobre la responsabilidad introducen a menudo un erróneo esquema causal o psicológico, quizá porque sospechamos que si (ciertos) factores, externos o internos, sobre los cuales no ejercemos control, son causa de que seamos como somos, se ve obstruida cualquier clase de contribución activa (por nucstra parte), a aquello que realizamos» (p. 2). Por supuesto los librealbedristas, desde siempre, no sólo creen que ese esquema causal es erróneo, sino que lo consideran la amenaza más sombría que haya podido cernirse sobre la libertad, y así lo han declarado, ininterrumpidamente, a través de los siglos. Pero filósofos como Galen Strawson (1989), en lo reciente, han dado en ver -muy elegantemente- que si bien el libre albedrío resulta imposible si el determinismo es cierto, también es imposible en cualquier otro caso. Y que todas y cada una de las amenazas que el determinismo parece suponer para nuestra concepción ordinaria de nosotros mismos como agentes libres, se darian por igual si supieramos que el determinismo es falso.

No obstante, tanto Strawson como los compatibilistas actuales que con anterioridad he citado concuerdan en afirmar que, si hemos de ser verdaderamente merecedores de elogio o de reproche por nuestros actos, entonces, $y$ dado que nuestras acciones intencionales están en función de lo que somos mentalmente, debemos también ser, de algún modo, responsables de eso que somos mentalmente; ser, al menos a ciertos respectos, verdaderos 'originadores' de nuestras naturalezas (algo que por lo demás Aristóteles, aunque en otro sentido, ya afirmo). Ello parece suminos, sin embargo, en una suerte de regressus ad infinitum, pues incluso si pudierámos escoger, ab initio, lo que queremos ser -como parecen pretender los libertaristas-, para llegar a serlo no sólo deberiamos existir ya, previamente, sino que habríamos de hacerlo provistos de ciertas preferencias que nos permitan elegir la clase de yo que vamos a querer ser. $Y$ si se nos ha de considerar responsables de csas preferencias, sin duda habriamos tenido que escogerlas, a su vez, con anterioridad, pero desde una instancia presumiblemente anterior, y así sucesivamente...

Con todo, el papel que juega el entendimiento en los intentos jerárquicos de redefinir el agente moral, que tienen en Frankfurt (1971, 1987) a su más notorio defensor, puede dar cuenta de ese cambio de interés por parte del compatibilismo del que hablaba al principio, tanto como del problema planteado por Strawson. No se trata de que el agente pueda hacer lo que quiera; ni siquiera de que pudiera hacer lo que habria decidido, si hubiera decidido de otro modo, sino de ser capaz de mantener deseos de segundo nivel, y voliciones de alto orden con las cuales nos identifiquemos. 
Asf pues, Frankfurt da un paso más que los librealbedristas, porque su concepción de la voluntad sf tiene que ver con "querer lo que uno quiere querer, o tener la voluntad que uno desean (1971 [1982, p. 90]), por decirlo con sus propias palabras. Si nuestra querencia particular se concreta de acuerdo con nuestras voliciones de más alto nivel, parece que entonces aseguramos, al fin y de una vez por todas, nuestro libre albedrío. Porque, al creer de Frankfurt, si nuestra volición es fruto de un deseo de segundo orden, es entonces fruto de la persona, y en la acción que resulte de esa volicion habremos actuado por propia determinación, o, lo que sería lo mismo, libremente.

Este intento jerárquico de resolver el problema de la responsabilidad puede enriquecer, a no dudarlo, las interpretaciones del sujeto propias del compatibilismo clásico. El yo debe ser entendido, así, en términos de voliciones de alto orden. Pero la concepción jerárquica del yo está sujeta a deficiencias insalvables, como Stump (1988), entre otros muchos, ha puesto en claro. Porque no es dificil objetar que una preferencia de alto orden puede ser tan defectuosa en un sentido moral, y estar tan fatalmente determinada, como lo son y lo están con frecuencia los deseos más irreflexivos, y esta crítica a la jerarquización es particulamente lúcida en los escritos de Susan Wolf (cf. 1987, 1990). Por lo demás, y como en breve argüiré, la decisividad del compromiso adquirido por el agente con esas voliciones de orden más elevado, sólo garantiza, a la postre, que la persona sea tal y como la caracterizan dichas voliciones - mientras dure su identificación con ellas, claro estáaunque también, y ésta es un arma de doble filo para la actuación moral, que el agente vea como externos a él los deseos que sean contrarios a ese sistema evaluativo desde el cual rige su actuación.

En ese sentido, la concepción de la acción libre y responsable, del modo en que se da, por ejemplo, en Watson $(1975,1987)$ difiere para bien de la de Frankfurt, tal y como Wolf (1987) ha puesto en claro. Watson identifica la clase de deseos propios del agente con deseos que son valores. Para Watson, si bien es cierto que el que una acción sea intencional depende meramente de la habilidad del agente para hacer que su voluntad promueva la conducta pertinente a cada volición, una acción libre consiste en poseer la más reflexiva habilidad de trasmutar los juicios de valor en volunlad efectiva (esto es, en querer -en sentido volitivo- aquello que consideramos bueno, sin la interferencia de motivos y circunstancias que obren en contra de esos valores meditados). Son deseos que en realidad consisten en la expresión de los juicios a través de los cuales el agente evalúa como bueno el objeto de su volición. En la medida en que sus acciones pueden ser gobernadas por esta segunda clase de deseos, el agente es libre, y de esas acciones puede considerársele responsable.

Pero si Watson ha aducido que el presunto estatuto del hombre-de-librevoluntad-en-el-mundo como un ser peculiar, es una parte crucial del problema del libre albedrío que puede ser solventado al vincular nuestros deseos más propios a la racionalidad, al cabo no llega más que a considerar ciertas condiciones últimas de la acción libre, como lo son - a su parecer- la plena intencionalidad y la ausencia de compulsion u obstrucción a la accion, requisitos ambos desafortunadamente compatibles con el hecho de que la tcoría del valor adoptada (esto es, el sistema de deseos 'racionales' en fun- 
ción de los cuales actuemos), sea producto de una perversa percepción de lo que realmente importa en el orden moral.

Charles Taylor (1976) por su parte, y al mismo respecto, afirma que quien "despliegue un lenguaje de contrastes evaluativos que se extiendan sobre los deseos, puede ser llamado un evaluador en scutido fuerte" (p. 287). Dicho evaluador "no define lo deseable solo por aquello que desea, o por aquello que desea más un calculo de consecuencias; (lo deseable) es definido además por una caracterización cualitativa de deseos como más altos o más bajos, más nobles o más indignos, y así sucesivamente..." (ibid.). Tal y como Wolf destaca (1987, p. 49), según Taylor unuestra libertad y responsabilicad dependen de nuestra habilidad para reflejar, criticar y revisar nuestros yoes». Pero, por lo demás, Taylor asegura que «no escogemos nuestras evaluaciones. Al contrario, éstas son articulaciones de nuestro sentido de lo que es digno, o más alto [...] (y) dar cierta articulacion es informar nuestro sentido de lo que deseamos o lo que consideramos importante, de cierta manera» (pp. 294-295). Ese 'informar nuestro sentido de lo que deseamos', debe subyacer a aquello que sea "de fundamental importancia para nosotros» (p. 296). Sin embargo, lo que importa para el caso es que el autocuestionamiento del yo a partir de la definición de sus más rudimentarias evaluaciones de sî mismo constituye lo esencial para la identidad del sujeto, aquello que permite, según Taylor, que le sea atribuible un estatuto de responsabilidad.

Todos estos filósofos, al ocuparse del tema de la responsabilidad, piensan -en la línea de Aristóteles- que las acciones, que son expresión del carácter del agente, son acciones con res- pecto a las cuales se le puede adscribir autoría, si es que aquél ha sabido reflexionar sobrc ese mismo carácter, esto es, si el agente cumple la condición de ser persona, algo que para Frankfurt significa —como hemos visto- poder mantencr voliciones de alto nivel que permitan la auto-identificación, y para Taylor la redefinición radical del sujeto (por si mismo) que se sigue de la naturaleza de un evaluador en sentido fucrte'. Pero, como veremos en lo que sigue, Wolf considera insuficientes estas condiciones de responsabilidad, a las que agrega otra, a su juicio mucho más importante. La definición de esa condición (por parte de dicha autora) nos llevará a un problema relativo, de quizá mayor importancia para un compatibilista -y que en todo caso tiende tout court a serlo-, a saber, el de cómo distinguir evaluativamente entre auto-reflexiones que conllevan actuaciones en el mundo imponderablemente dispares desde el punto de vista de la moralidad, y tras ello, el de cómo decidir la ventaja moral de ciertas determinaciones-sobre-nosotros-porparte-del-mundo en contraposición a otras...

\section{II}

Como bien lo ve Wolf, la perspectiva del yo profundo -así la llama ellade los autores antes citados, puede, al cabo, transformar los miedos de aquellos que creen que el determinismo representa una amenaza para la acción libre y responsable, pero no erradicarlos. Porque a la postre las teorías de Frankfurt, Watson o Taylor no hacen más que distinguir entre los deseos que el agente quiere considerar como externos a él, y los deseos que sí son aceptados por el propio yo, el yo profundo. La cuestion que aterra a los anti-deterministas sigue, pues, irresuel- 
ta, porque cabe preguntarse qué cosa gobierna el sistema de valores del yo profundo, desde qué instancia se ven validados esos valores. Parecería, en fin, que sigue siendo cuestion de fortuna que el yo profundo dependa de nosotros, esto es, que nuestros juicios y valores más meditados y reflexivos no sean, por ejemplo, crróneos en virtud de un desafortunado aleccionamiento de la racionalidad, y que la justificación de esos valores a través de la mayor o menor profundidad del yo se perpetre adecuadamente. Pero por supuesto los téricos de la racionalidad, desde Aristóteles - que, por cierto, no tuvo que recurrir a niveles profundos del yo ni siquiera al tratar el tema de la incontinencia o de la akrasia- a Spinoza, habrian considerado o considexaron incomprensible la pretensión de que la asignación de responsabilidad requiera del agente que éste se halle en un punto de partida absolutamente inicial, porque es evidente que no podemos auto-crearnos, ni en el orden de la volición ni de la reflexión moral.

Podemos - los más afortunadosreplantear nuestra vida, auto-criticar las propias disposiciones, intentar incluso modificar nuestro carácter, de modo que lleguemos a ser libres no solo de hacer lo que queramos, y conseguir que los descos que inevitablemente tenemos puedan ser suspendidos como motivadores de la acción -a partir de otra instancia más apremiante- sino también de actuar a partir de lo que deseamos desear. Pero aunque así sea, no es ésta, pace Frankfurt, "toda la libertad que es posible desear o concebir» (1971, p. 16). Desde el punto de vista moral, se requiere de una libertad mucho mayor, tras la que Wolf (1987) va en busca.

Su contraejemplo, en el artículo en cuestión, es verdaderamente ilustrati- vo: "JoJo es el hijo favorito de Jo El Primero, un dictador sádico y malvado de un pequeño pais subdesarrollado. Dados los especiales sentimientos del padre para con el hijo, a JoJo se le da una educación especial, de modo que se le permite acompañar a su padre, y ser espectador de su actividad diaria. A la vista de ese tratamiento, no es sorprendente que el pequeño JoJo tome a su padre como modelo del rol (a realizar), y que desarrolle valores muy similares a los de papá. Ya de mayor, JoJo hace muchas de las cosas que hacía su padre, mandando incluso a la gente a cámaras de tortura o a la muerte, sólo por extravagancia. No se ve coercionado a realizar esos actos, [sino que] actúa según sus propios deseos. Más aún, se trata de deseos que desea plenamente tener. Cuando da un paso atrás [en la reflexión] y se pregunta: "¿Deseo realmente ser esa clase de persona?", su respuesta es, resueltamente: "Sf", pues este modo de vida es expresión de una suerte de poder desmesurado que forma parte de su más alto idealn (1987, pp. 53-54).

Hasta aqui la historia de JoJo. Dadas las circunstancias de su niñez, y su limitada experiencia, reducida a observar el comportamiento de su padre, sin poder contrastarlo con otra clase de tendencias en lo que se refiere a la actuación para con los demás, podría dudarse -opina Wolf- de que JoJo sea responsable de lo que hace. Arguye la autora: "No está claro que cualquier otro, con esa infancia, pudiera haberse convertido en otra cosa que en la clase de persona perversa y torva que (JoJo) ha llegado a ser» (ibid.). Y sin embargo, los deseos de JoJo son gobernados por su yo más profundo, porque no pademos decir de el que, en tanto que agente, no es el yo que desea ser, o que no realiza las acciones que (verdaderamente) desea realizar. JoJo, en st1 
interior "se siente tan integro, libre y responsable» (ibid., p. 54) como podemos sentirnos nosotros en los momentos de más plena autoaceptación.

Desde nuestra perspectiva evaluativa, puede parecer que JoJo no es libre, en el sentido de que su yo más profundo no depende de $e l$, ni ha sido decidido por él, y en efecto asf es. Pero, si observásemos nuestros propios yoes desde el exterior, veriamos que tampoco éstos dependen de nosotros, en el mismo sentido y por razones idénticas. Así, podemos dudar de que nuestro yo más profundo dependa de nosotros, como sabemos que no lo hacen los más intolerables de nuestros deseos inmediatos. Pero el problema que ello suscita es independiente de la verdad del determinismo. Porque ya sea que estemos determinados causalmente o no lo estemos, nos es imposible crear nuestros yoes más profundos. Así que si Jolo no es responsable, puesto que su yo-más-profundo - con el que está, no lo olvidemos, plenamente de acuerdo desde cualquier otra instancia- no le pertenece, tampoco nosotros podemos serlo. ¿Ocurre que debemos ejercer otro tipo de control sobre nuestros yoes más profundos, que nos permita asumir una plena responsabilidad por lo que hacemos? $\mathrm{Y}$ si es asi, ¿desde qué instancia hacerlo? Pero no, no es asf. Porque como Wolf afirma con acierto: «No todas las cosas necesarias para la libertad y la responsabilidad deben ser tipos de poder y control [...] Puede que necesitemos, simplemente, ser de cierto modo, incluso si no está en nuestro poder determinar el que seamos así o now (p. 55).

A juicio de Wolf, una condición crucial para la responsabilidad, a la que se refiere la observación anterior, supone que el agente que realiza la accion debe estar cuerdo ('an agent 'must be sane). Como ya he dicho, no esta en nuestro poder determinarnos a estarlo o no; no lo está tan siquiera cumplir las condiciones iniciales para poder hacerlo (algo que, por lo demás, Spinoza afirmó tajantemente ["no está en poder del hombre tener una mente sana más de lo que puede estarlo tener un cuerpo sano", TP 2/6, Ep. 78], y que los griegos parccieron sospechar, dado su terrible respeto hacia la týche). Podrá decirse, pues, que somos afortunados si lo conseguimos.

Pero el 'estar cuerdos' no significa que ejerzamos una suerte de control sobre nuestras acciones que los nocuerdos' no podrían ejercer. Según Wolf, se trataría más bien de aleccionarse a uno mismo, de modo que las propias percepciones y razonamiento nos comprometan con una concepción del mundo que se revele como pertinente a la libertad. Lo cual no requiere tanto de un control previo sobre la voluntad y las acciones consecuentes, sino que, bien al contrario, garantiza que las acciones realizadas por el agente concuerden o se sigan naturalmente de su correcta comprensión del mundo. La cordura sincluye todo aquello que nos capacita para desarrollar una concepción adecuada del propio mundo» (1987, p. 55, nota), y se precisa para ello de la sensibilidad y de la experiencia emocional tanto como del razonamiento ( la sensibilidad y la exposición a ciertos reinos de experiencia pueden también ser necesarios para tal fins, ibid.).

Lo realmente importante del analisis de Wolf es que da cuenta de cómo los valores particulares de la gente, por meditados y coherentes que sean entre sí, pueden ser inapelablemente erróneos, y en dicho sentido la libertad de la voluntad, que permite a los agentes realizar acciones con acuerdo a dichos valores será, en principio, algo que puede obrar en contra de la cordura 
moral. Dire, sin embargo, que aunque percibo claramente la diferencia entre la libertad defendida por Frankfurt o Watson (que es en definitiva la libertad compatibilista acerca de la acción libre), y el libre albedrío (Hill [1984] es un artículo esclarecedor al respecto), pienso que la libertad propugnada por Wolf en el artículo de 1987, al poder adivinarse como metafísica, es en esc sentido una alternativa idónea a la posición libertarista, porque la adecuada percepción del mundo que propugna (y que promovería los valores que motivan nuestras acciones), tendría a la vez el valor de trasmutar la propia voluntad, de modo que ésta no pueda no querer actuar en el sentido que le impone dicha percepción, si es que ocurre que percibir el mundo de manera adecuada nos imponga una actuación determinada en él, en contraposición a otras.

Por supuesto, y tal como la misma Wolf observa, una réplica inmediata cuestionaría el modo en que un yo puede saber que está más cuerdo que otros por el hecho de que entiende el mundo de cierta manera. $Y$ es aquí donde la autora se equivoca aparatosamente, cuando observa que snada justifica (una determinada concepción del mundo y de los valores) excepto el acuerdo intersubjetivo extenso, y el [...] éxito que obtengamos al divulgarnos en el mundo y satisfacer nuestras necesidades" (1987, p. 60). Porque afirmar lo anterior es caer, al final, en el mismo relativismo contra el que se luchaba. ${ }^{A}$ qué clase de éxito se refiere Wolf? [...] Sin duda, y a juzgar por el mucho tiempo en el que han permanecido en el poder, muchos dictadores parecen haber tenido sobrado éxito-enel-mundo con el sistema de valores que adoptaron, y sin duda se divulgan más - tienen más repercusión, en la mayoría de los casos-, las acciones aberrantes que otras más razonables. Caligula, por ejemplo, satisfizo sus necesidades con más frecuencia que otros que $\sin$ duda estuvieron más cuerdos que él, y, en fin, un amplio acuerdo intersubjetivo está en la base de teorías sociales y de la justicia que no todos calificariamos de sensatas. Cabe pues convenir en que Wolf plantea esta cuestión crucial para la moralidad de manera precisa y elegante, pero no llega a ver que la solucion que propone para la misma pervierte al final toda su reflexion. Porque aunque en un principio nos deja sospechar con alivio que su concepción de la libertad no es nomativa, al final impone para la misma pautas como el acuerdo colectivo o la adaptabilidad individual, elevándolas al rango de criterios de justificacion.

\section{III}

En 1990 aparece Freedom within Reason, libro en el que Wolf entiende dar respuesta a ciertos de los interrogantes dejados en suspenso en el texto de 1987. Porque si se arguye que estar cuerdo -y ser, por lo tanto, responsable-conlleva ese deseo de que la percepción que tenemos del mundo se concrete en un entendimiento adecuado de lo que sea correcto o incorrecto, Wolf debía aún dar cuenta de ciertas cuestiones, v.gr.: ¿De qué manera tenemos que ser? a ¿cómo saber que nuestra comprensión del mundo es adecuada o no, sin caer en el mero consecuencialismo? [...] Más allá de las voliciones de alto nivel de Frankfurt (duramente criticadas por Wolf en el capítulo 2 de su libro), o de la capacidad para evaluar los deseos, que Taylor reconoce como constitutiva de la responsabilidad, el criterio de cordura wolfiano parecía prometer una estricta diferenciación entre agentes responsables 
e irresponsables que, levantándose sobre la correcta comprensión del mundo (a través de la cual se definen los primeros) explicaría su posterior actuación como-agentes-cuerdos-en-el. Lo hemos visto: "podemos entender la cordura [...] como la mínima habilidad suficiente - tanto cognitiva como nomativamente- para reconocer $\mathrm{y}$ apreciar el mundo por lo que es» (1987, p. 56).

Así pues, ¿qué es el mundo?... Wolf (1987) supone que saberlo y apreciar ese conocimiento es cuestion de habilidad, y afima al respecto que un agente moral puede reconocer como erroneas, desviadas o pérfidas ciertas acciones $\mathrm{en}$ base a un entendimiento del mundo por lo que es, sin dar, sin embargo, una justa respuesta a la cuestión esencial... ¿Qué es el mundo? Si lo supiéramos, serfamos capaces -siempre según Wolf - «de entender y evaluar nuestros caracteres de modo razonable» (1987, p. 59), además de gozar de la ulterior habilidad "de corregimos a nosotros mismos», más allá de la verdad o falsedad del libre albedrio como posibilidad metafísica, $o$ de nuestra creencia o no en un determinismo ferreo por cuanto se refiere al modo en que hemos alcanzado esa comprensión.

El criterio wolfiano para deslindar la cordura de lo que no lo es puede parecer infrecuente, y aun irreal. Ciertamente lo es. Más allá de la habitual exigencia de eximir de responsabilidad a aquellos que consideramos locos, y de que sin duda éstos muestran una patente desconexión con respecto a ciertos intereses mundanos (desconexión que no abunda entre los demás), nada parece indicar que, sin embargo, lo contrario - «una correcta comprensión del mundo, tanto cognitiva como normativamente" - sea una evidente y constante capacidad de quienes son te- nidos, en general, por cuerdos. Con ello quiero decir que la habitual habilidadpara-actuar-correctamente-en-el-mundo no parece requerir ningún tipo de compromiso ontologico (de conocimiento y apreciación de lo que el mundo sea) que garantice la responsabilidad moral de un agente cuerdo relativa a sus acciones, sino tan sólo una suerte de certidumbre acerca de uno mismo como causante directo de la acción, certidumbre de la cual no podemos asegurar que JoJo, en el ejemplo de Wolf, carezca.

El planteamiento que Wolf propone de la misma cuestión en 1990 es algo diferente. Se tratarfa de saber cómo pueden las personas merecer un elogio real o profundo por ser, por ejemplo, generosas o amables, más allá del que puede merecer una rosa por ser escarlata o un colibrí por volar velozmente. $Y$ si la posición que basa la responsabilidad en la autonomía tiene como principal finalidad dar respuesta a dicha cuestión, lejos de hacerlo - arguye Wolf- esa concepción del libre albedrío como indiferencia anula la posibilidad de que las personas merezcan el elogio por las cualidades mencionadas (o por cualquier otra), porque sería la voluntad libre incondicionada la que lo merecería y no el agente, y todo libertarista tiene difícil probar que la voluntad se corresponde absolutamente con la persona, en la medida en que afirma que el carácter, los motivos o las creencias del agente (esto es, aquello que constituye su identidad) en nada comprometen a la voluntad cuando el yo elige libremente. De modo que la tesis según la cual un agente responsable debe ser autónomo, esto es, capaz de hacer lo que efectivamente hace y que a la vez otras (al menos otra) posibilidades estén abiertas para el, en sentido metafísico (es decir, que sean alternativas reales 
en el mismo momento y en idénticas circunstancias) es refutada por Wolf en el capítulo 3 de su libro. El gran error de la concepción de la autonomía como independencia-con-respectoa-las-causas es, según la autora, que equivale a mantener que lo que importa no es cuál sea la acción que el agente decide realizar o realiza de hecho (para atribuirle responsabilidad), sino $\tan$ sólo que haya podido hacer otra cosa que la que efectivamente hace. Pero Wolf niega que la responsabilidad tenga que ver con esas posibilidades alternativas, las cuales nada garantizan de la cordura o sentido moral del agente, ni de la calidad en fin de su elección. En contra de la posición libertarista, Wolf piensa que «lo que importa cs, más bien, la accesibilidad (availability) de una opción muy particular. La opción de actuar de acuerdo con la Razón" (1990, p. 68) (como es de notar, Wolf ha modificado la terminología mantenida en su artículo de 1987, donde hablaba de cordura para referirse a las condiciones necesarias para la responsabilidad. Veremos que esta Razón que proclama ahora como criterio de evaluación de las acciones no la aleja mucho, con todo, de aquella concepción).

Hasta tal punto es eso lo importante que, si un agente actúa con acuerdo a la Razón, será irrelevante el hecho de que haya podido o no actuar en desacuerdo con ella. Aunque si, inversamente, el agente no ejercita esa opción, entonces wla cuestión de si hubiera podido hacerlo o no resulta extremadamente importante» (ibid, p. 68). Mientras que el defensor de la autonomía insiste en que el agente debe ser capaz de escoger y actuar de modo que no se haya visto obligado por las características incontrolables de su modo de ser, o por los eventos del pasado, Wolf propone esa 'Perspectiva de la Razón' (the Reason View), según la cual toda la flexibilidad que requierc una elección moral es que el agente sea capaz de actuar de acterdo con la Razón.

Parte de lo que comporta elegir según la razón es, en opinión de Wolf, ser sensitivo y responder a los cambios relevantes del entorno y de la situación particular del agente. Queda claro, pues, que el paso desde el criterio de autonomía al de la Razón no es insignificante. Porque la idea de que se requiere autonomía para gozar de libre albedrio y responsabilidad es claramente metafísica, se refiere a una independencia-con-respecto-a-las-causas que permita la auto-causación en el orden de la voluntad. Pero según Wolf la cuestión de saber si somos libres y responsables no concierne a la clase de entidades metafísicas que somos. La Razón es la facultad que nos posibilita formar creencias verdaderas y buenos valores, y esa habilidad de pensar correctamente confirma nuestro saber cómo es el mundo y la certeza de que no estamos equivocados en ese entendimiento. "Debemos saber si tenemos la habilidad de escoger y actuar sobre la base de las razones correctas para elegir y actuar. La cuestión de si tenemos esa habilidad no es metafísica, sino metaética, o (meramente) ética. Se debe dar respuesta a ella sólo tras saber que sería hacer lo correcto y tener las razones correctas para actuar" (Wolf 1990, p. 71).

Tener un estatuto de responsabilidad sería el poder de ejercitar la Correcta Razón, y gobernar las propias razones a través de ese ejercicio, lo que Wolf describe como sla capacidad de reconocer lo Verdadero y lo Bueno» (ibid.). Pero ser responsable requiere no sólo de esa habilidad del agente para actuar de acuerdo con lo Verdadero y lo Bueno, sino que lo haga por mor de ello. $\mathrm{Y}$ 
de algún modo -afirma Wolf-, la persona que es capaz de ejercer esa habilidad no se halla gobernada por el pasado, aun si ocurre que nos hallamos en un mundo férreamente determinista, porque la Razón-como-Agente (en contraposición a la Voluntadcomo-Agente de los libertaristas), al erigirse como causa de la acción, la origina. Así pues, ser 'metafísicamente corriente' (esto es, actuar en el mundo de un modo no-excepcional con respecto a las leyes de la naturaleza) es suficiente y compatible con el hecho de ser libre. Con todo, Wolf debería probar que, puesto que queremos ser responsables de lo que hacemos (y responsables nos sentimos con respecto a una gran mayoría de las acciones que realizamos), todos gozamos en lo habitual de esa habilidad de reconocer lo Verdadero y lo Bueno, y de actuar de acuerdo con ello, y en virtud de ello.

Por otro lado, si lo que importa no son las posibilidades alternativas, sino la calidad de la acción, deberá existir un baremo objetivo de evaluación de las diversas calidades de lo que hacemos, o en la teoria de Wolf ( $y$ el redundar se hace obligado), una prueba de que nuestro entendimiento de lo Verdadero - tanto cognitiva como normativamente - se ajusta a la verdad. ¿Cuál es el modo en que el agente aprehende lo Verdadero y lo Bueno, en sus juicios de valor? Wolf se limita a afirmar que el hacerlo es una cuestion no sólo de inteligencia, de capacidad de observación o de complejidad psicologica. Porque una persona con esas cualidades pero que ha sufrido un estricto aleccionamiento de motivaciones perversas (como JoJo) puede sufrir una irremediable distorsión de sus valores sin ser, pese a ello, inhábil para integrarse en el mundo. Un agente responsable, inversamente, debe haber tenido experiencias que le permiten esa apreciación del mundo por lo que es, que en opinión de Wolf le permite discernir lo Verdadero y lo Bueno, pero además ese discernimiento habra de ser el motivador de la acción. Puede que se necesite, al respecto - apunta la autora- haber desarrollado una sensibilidad especial hacia los buenos valores, y gozar de una disposición correcta hacia el mundo, en virtud de la cual la atención, la simpatía, el entendimiento y el razonamiento del agente sean fructiferos (surtan efecto, podríamos decir). A la luz de esa compleja habilidad, y merced a ella, un agente puede actuar correctamente, y rcalizar su acción por las razones correctas.

Pero si un agente es incapaz de hacer lo correcto por las razones correctas, entonces no es culpa suya el sucumbir, al realizar algo incorrecto» (ibid., p. 81). Hallamos aquí, de nuevo, la asimetría a la que Wolf se referia ya en un cclcbrado artículo de 1980, asimetría que en la historia de las ideas puede rastrearse - a través de la Reforma- hasta la concepcion agustiniana de nuestra inevitable tendencia hacia el mal si no nos vemos ayudados por una facultad salvífica (secularizada e internalizada, por supuesto, en el caso de Wolf). Quede claro, en todo caso, que en la teoría wolfiana no es el inevitable ejercicio de la Razón lo que nos hace responsables, sino sólo la habilidad de realizarlo, que la autora parece suponer ampliamente extendida. La idea de Wolf, correcta en mi opinion, es que hay algo liberador en nuestro entendimiento correcto de lo que es el mundo, y que si la Razón sabe que no se equivoca en ese entendimiento, el agente -en consecuencia- obra correctamente. Por ello una persona incapaz de actuar a partir de las razones adecuadas debería ser compadecida antes que culpada. Y no obstante queda sin resolver una cues- 
tión capital: ¿cuáles son las razones adecuadas para actuar correctamente en el mundo? Wolf insiste sin descanso en la importancia de actuar de acuerdo con lo Verdadero y lo Bueno, pero no nos ayuda a discernir que sea ello. Y si el criterio que distingue entre agentes responsables y quienes no lo son carece de consistencia sustantiva, puede objetársele a la autora que, considerándolo en términos subjetivos, pocos dejan de actuar con acuerdo a ese criterio.

Algo existe objetivo que permite dilucidar las razones correctas para actuar, y nos las hace accesibles. Wolf parece intuirlo pero no acierta a definir o describir ese vínculo, de modo que cae en el mero consecuencialismo, afirmando de nuevo que querer escoger a la luz del conocimiento de lo Verdadero y lo Bueno es meramente «querer ser capaz de escoger a la luz del conocimiento de las opciones que se nos presentan y de las razones comparativas en pro y en contra de esas opciones (1990, p. 92). Pero por desgracia, las personas voluntariamente inmorales han decidido con frecuencia realizar sus perfidias con acuerdo a ese conocimiento, porque entienden, por ejemplo, que el mundo es de tal modo que cualquier condicionamiento moral podrfa definirse como contranatura. Sade fue un maestro en exfoliar esa perfecta coherencia entre un entendimiento razonado del mundo y la más abyecta criminalidad. Es más, un entendimiento de cómo es el mundo está en la base (es la motivación) de las aberrantes acciones de sus libertinos, que gozan de todas las habilidades que Wolf atribuye a los agentes responsables (sensibilidad, capacidad de atención y de reflexión) en un grado quizá mayor del que puede suponerse que gozamos en general los humanos. Wolf debería, quizá, releer a
Sade para darse cuenta de que su teoría requiere de mucho mayor contenido sustantivo, en lo que se reficre a la cuestión capital: ¿qué es el mundo?, si quiere probar que actuamos por las razones correctas al aprehender lo Verdadero y lo Bueno.

Puede arguiirse pues, en contra de Wolf, que actuar por las razones corectas podría significar, en el mundo, comportarnos de manera inmoral. "Comprender las características significativas de la propia situación y las alternativas entre las cuales podemos elegir» puede fácilmente conducirnos, pace Wolf, a alejamos de lo Bueno, si no de lo Verdadero, mientras no se pruebe lo contrario. $Y$ puesto que en la mayoria de casos creemos saber que actuamos por las razones correctas, la certeza real a tal respecto debe tener un origen más fundamental que todo eso. Empeñarse en afirmar que ver y apreciar lo Verdadero y lo Bueno comprende una correcta y suficientemente amplia apreciación de principios normativos requiere de una sólida y reveladora justificación, que Wolf no nos da. Porque decir que ver el mundo correctamente comporta darnos cuenta de lo que es valorable y lo que no lo es, pero afirmar también que actuamos correctamente y por las razones correctas al apreciar el mundo por lo que es, no hace más que encerrarnos en un círculo vicioso inexorable.

En lugar de dar cuenta de la cuestion capital (¿Qué es el mundo?), Wolf dedica el resto de su argumentación, casi patéticamente, a defenderse de la acusación de objetividad que sospecha que podría levantarse contra su teoría: "los supuestos metaéticos necesarios (para defender mis tesis acerca de la responsabilidad) relativos a la objetividad son de hecho perfectamente débiles y extremadamente plausibles» (1990, p. 118). Su concepción de la responsabilidad es 
compatible, en opinión de la autora, con wuna gran variedad de posiciones metacticas" (ibid.). Sin embargo, Wolf confiesa que una de cllas le parece más plausible que las otras, desde la Perspectiva de la Razón'.

$Y$ ésta es: que la percepción, imaginación, reflexión, instrucción, y los poderes lógicos del pensamiento «son relevantes para [...] ciertos métodos aceptables de llegar a poder formular juicios de valors (pp. 123-124). Gracias a ello, el agente responsable puede demostrar 'competencia normativa' en su actuación, lo que comporta, de algún modo, Ia existencia de patrones de corrección no arbitrarios, «independientes de la voluntad del individuo, e incluso de la psicología del individuo como un todo, mediante los cuales uno puede juzgar algunas acciones, elecciones, modos de vida o sistemas de valor como mejores que otros" (ibid, 124). Con todo, esos patrones no determinan wun sistema de valores único, universalmente aplicable, completo y óptimos (ibid.) -añade la autora- puesto que las razones que existen para cl agente, en un momento dado, pueden no determinar que una elección concreta sea mejor o más correcta que otra, y la persona será responsable en la medida en que es capaz de hacer los mejores juicios de valor dadas esas razones.

De modo que Wolf defiende lo que ella llama un pluralismo normativo, según el cual los valores y juicios de valor son sólo parcialmente objetivos, algo que permite una variedad de perspectivas morales sustantivas. "La 'Perspectiva de la Razón' asocia la libertad y la responsabilidad del agente con la habilidad de entender y apreciar qué razones se dan" (p. 140), y esas razones "pueden provenir de una gran variedad de fuentes, no necesariamente todas conmensurables, ni capaces de provocar las mismas decisiones en todos los casos" (p. 141). Pero, ¿cómo puede Wolf afirmar lo anterior, sin haber dilucidado previamente qué apreciacion del mundo nos permite el ejercicio o la simple posesión de esa habilidad para actuar de acuerdo con lo Verdadero y lo Bueno, esto es, por las razones correctas? Mi idea sobre esta cuestión es opuesta a la de Wolf, porque aunque ella afirma con acierto que se requiere de un entendimiento de lo que el mundo sea (para actuar teniendo como causa las razones correctas), a la pastre son sola las experiencias de los agentes más afortunados (un dichoso aleccionamiento de la sensibilidad y la capacidad de reflexión sobre las circunstancias) las que les permiten una mejor o más aguzada comprensión de cómo es el mundo, pero no de lo que es. Y si lo que sea el mundo sigue siendo la cuestión capital, esa cuestion requiere, pace Wolf, de una consideración ontológica fuerte, que dé cuenta además de nuestro estatuto como-seres-racionales-y-volitivos-queactuan-en-el (bien lo vio Spinoza al iniciar su Ethica -en la que anticipa, sea dicho en passant, las más afortunadas de las tesis wolfianas- con una demostración de lo que el mundo sea [Deo lo llamó, en una terminología forzada por los tiempos]). Wolf debe admitir, así, una terrible carencia en su 'Perspectiva de la Razón', la de que desde su teoría no se atisba siquiera una solución al problema de encontrar cl modo en que podríamos determinarnos a ver el mundo como lo que es, y esa es la cuestión capital, a mi parecer, en lo que se refiere a la libertad. Sin esa solución, su misma aceptación de que estar determinado es compatible con la Perspectiva de la Razón resulta, además, temiblemente peligrosa, en la medida en que nos vemos en la tesitura de tener que accptar que, si no lo- 
gramos saber actuar por las razones correctas, estar determinados en otro sentido nos aboca fatalmente a ser irresponsables y a carecer de libertad. Es claro que en ningún caso cabría esperar udesembarazarnos de todas las influencias que están potencialmente en conflicto con la razóns (p. 146), pero de una teoría que pretende erigirse como alternativa al libertarismo querriamos al menos poder esperar que se nos desvelara como hacerlo, siquiera en los casos en los que de ello dependan nuestra libertad y responsabilidad como agentes-volitivos-enel-mundo.

\section{REFERENCIAS}

ARIstoreles: The Works of Aristotle (trad. inglesa de W.D. Ross), tomos I-XII, Oxford University Press, 1988.

Benson, Paul (1987): "Freedom and values, The Joumal of Philosophy, 84, 465-486.

Chisholm, Roderick M. (1967): He Could Have Done Otherwises, The Joumal of Philosopy, 64, 409.417.

Denner, Daniel C. (1984a): Elbow Room, Mit Press and Oxford University Press.

-(1984b): "I Could not Have Done Otherwise. So what? , The Joumal of Philosophy, 81, 553-565.

FRANKFuRT, Harry (1969): "Alternate Possibilities and Moral Responsibility*, The Joumal of Philosophy, 56, 829-839.

- (1971): «Freedom of the Will and the Concept of a Person, The Jounal of Philosophy, 68, 520; reimpr. en Gary Watson (ed.), Free Will, Oxford University Press, 1982.

- (1987): kIdentification and Wholeheartedness". en F. Schoemm (ed.), Responsibility, Character and the Enotions, Cambridge University Press. $27-45$.

HrL, Christopher S. (1984); "Watsonian Freedom and Freedom of the Will», Ausmalian Jounal of Philosophy, 62, 294-298.

Hosbes, Thomas (1966): English Works (ed. W. Molesworth), 11 vols., Nueva York.

Hofstadter, Douglas R. (1979): Godel, Escher, Bach: An Etemal Golden Braid, Basic Books Inc-; trad. cast. Gödel, Escher, Bach. Un etemo y gracil bucle, Tusquets.

- (1982): «Who Shoves whom around inside the Careenium? of What is the Meaning of the Word 'T?

MOORE, George (1912); Ethics, Londres.
Schoeman, Ferdinand (1987): entroductions, en F. Schoeman (ed.), Responsibility, Character and the Emotions, op. cit., 1-24.

SrRaWson, Galen (1986): Freedom and Belief, Oxford, Clarendon Press.

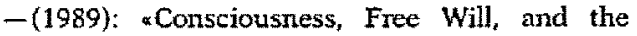
Unimportance of Determinismn, Inquiry, 32 , 3-27.

STump, Eleonore (1988): «Sanctification, Hardening of the Heart, and Frankfurt's Concept of Free Will, The Joumal of Philosolpy, 85, 395420.

TAYLoR, Charles (1976): «Responsibility for Self", en A. Rorty (ed.). The Identities of Persons, University of California Press, 281-299; reimpr. en G. Watson (ed.), Free Will, Oxford University Press; 1982.

VAN INWAGEN, Peter (1983): Anz Essay orz Free Will; Oxford University Press.

Watson, Gary (1975): "Free Agency», The Joumal of Philosophy, 72, 205-220.

- (1987): Free Action and Free Will*, Mind, 96, 145-172,

Worf, Susan (1980): «Asymmetrical Frecdom*, The Joumal of Philosophy, 77, 151-166.

- (1981): «The Importance of Free Will», Mind. 90, 386-405.

- (1982); "Moral Saints", The Journal of Philosophy, 79, 419.439.

- (1986): above and Below the Eine of Duty*. Philosophical Topics, 14, 131-148.

- (1987): * Sanity and the Metaphysics of Responsibility», en F. Schoeman (ed.), Responsibility, Character and the Emotions, op. cit., 46-62.

- (1990): Freedom within Reason, Oxford University Press. 